\title{
A comparative study of Candida species diversity among patients with oral squamous cell carcinoma and oral potentially malignant disorders
}

\author{
Sankar Leena Sankari ${ }^{1}$, Krishnan Mahalakshmi ${ }^{2^{*}}$ (10 and Venkatesan Naveen Kumar ${ }^{3}$
}

\begin{abstract}
Objectives: To determine the prevalence of Candida species by PCR-RFLP method in the saliva of patients with oral squamous cell carcinoma (OSCC), oral potentially malignant disorders (OPMD) and healthy cohorts. Unstimulated saliva was collected from patients with OSCC $(n=97)$, OPMD $(n=200)$, and healthy controls $(n=200)$. Candida species were isolated using the standard protocol. The isolates were identified using phenotypic and genotypic methods. The odds/risk ratio was calculated using Pearson's Chi-square test. The significance of Candidal carriage was calculated by independent T-test.

Results: Oral Candidal carriage was 72.2\%, 58\% and 20.5\% among patients with OSCC, OPMD, and healthy controls respectively. The oral Candidal carriage in OSCC and OPMD was highly significant ( $p=0.0001)$. Non albicans Candida predominated over Candida albicans. Candida species were diverse among the study groups with a predominance of Candida krusei, Candida tropicalis, and Pichia anomala formerly Candida pelliculosa. P. anomala occurrence outnumbered in health. The odds/risk ratio for OSCC and OPMD were 4.25/11.87 and 3.52/6.99 respectively. A high prevalence of non albicans Candida was observed both in all the three groups (OSCC, OPMD and healthy controls). High odds and risk ratio associates Candida species to OSCC and OPMD. Candida famata may be associated with OSCC and OPMD.
\end{abstract}

Keywords: Candida, Oral squamous cell carcinoma, Oral potentially malignant disorder, Polymerase chain reaction, Restriction fragment length polymorphism

\section{Introduction}

Globally oral squamous cell carcinoma (OSCC) is the foremost cause for mortality. OSCC is the sixth most common cancer reported globally with an annual incidence of over 300,000 cases. It is the 12th most common cancer in women and sixth in men. About $62 \%$ of the cases arise in developing countries. In South-central

\footnotetext{
*Correspondence: kmagvenkat@gmail.com

2 Department of Microbiology, Research Lab for Oral-systemic Health,

Sree Balaji Dental College and Hospital, Bharath Institute of Higher

Education and Research, Chennai 600100, Tamil Nadu, India

Full list of author information is available at the end of the article
}

Asia, it is the third most common type of cancer. In South and South East Asia, high incidence of oral cancer is found in Sri Lanka, India, Pakistan and Taiwan [1]. The age-adjusted rates can vary from over 20 per 100,000 populations in India, to 10 per 100,000 in the United States, and less than 2 per 100,000 in the Middle East [2].

OSCC are most often preceded by clinically apparent oral potentially malignant disorders (OPMD).The presence of Candida species in oral leukoplakia has strengthened its association to with OSCC as well [3]. Candida species have been linked to etiopathogenesis of OSCC and oral potentially malignant disorders 
considering its potential to nitrosylate $N$-benzyl methylamine $[4,5]$.

Candida are oral commensals, wherein it gains a precedence when defence mechanisms are compromised or there is a change in local oral microenvironment that is conducive to Candida growth such as low oxygen, low $\mathrm{pH}$ and an anaerobic environment leading to a diverse group of infections [3]. Changes in the epithelium with the presence of Candida may predispose to Candidal infection which may induce epithelial dysplasia and malignancy [5]. Leukoplakia with Candidal infection has a higher rate of malignant transformation than uninfected leukoplakia [6]. Lesions infected with Candida have shown progressively more severe dysplasia compared to patients without Candidal infection [5].

With considerable evidence associating Candida with OSCC \& OPMD, the present study aims to assess the prevalence of different Candida species among patients with OSCC, OPMD and to compare with normal healthy controls by genotypic methods (PCR-RFLP).

\section{Main text \\ Methods}

Study Design: The present study compares the prevalence of Candida species in the saliva of OSCC and OPMD patients with healthy subjects by phenotypic and genotypic methods. The period of recruitment was between December 2014 to June 2019 Study population: The study population comprised of patients with oral squamous cell carcinoma $(n=97)$, Oral potentially malignant disorders $(\mathrm{n}=200)$ and healthy controls $(n=200)$. The patients with OSCC \& OPMD were recruited from outpatient clinic, Department of Oral Pathology and Microbiology and Centre of Oral Cancer Prevention, Awareness and Research, Sree Balaji Dental College and Hospital. Oral hygiene was assessed using Oral hygiene Index-Simplified (Greene \& Vermillion 1964) and categorized as poor, fair, or good [7]. Subjects with poor oral hygiene were not included in the study as poor oral hygiene is considered principal risk factor for candidal colonization. Patients before any form of treatment and willing for biopsy were included for the study group. All the patients in OSCC \& OPMD group had habits of smoking/tobacco chewing along with alcohol consumption. Patients with uncontrolled diabetes, immunosuppression, denture wearers, poor oral hygiene status and patients receiving steroid or antibiotic, antifungal therapy for past three months were excluded. Age and gender matched subjects with no history of systemic diseases, oral mucosal lesions, and deleterious habits were recruited for the control group.

\section{Saliva collection and determination of colony forming units/ml}

Unstimulated whole saliva was collected by the "draining" method. The subject's head was tilted forward so that saliva moved toward the anterior region of the mouth and the pooled saliva $(2 \mathrm{ml})$ was collected into a wide-mouthed sterile container [8]. The sample was then immediately transported to the microbiology laboratory for isolation and identification of Candida species. $10 \mu \mathrm{l}$ of saliva was inoculated onto Sabouraud's dextrose agar (SDA) plate and incubated at $37{ }^{\circ} \mathrm{C}$ for 1 week. The purity was checked by Gram staining. Colony count was performed by a digital colony counter and expressed as colony forming unit $(\mathrm{cfu}) / \mathrm{ml}$ of saliva.

\section{Identification of Candida species Phenotypic methods}

The identification of Candida species was done by standard phenotypic methods (CHROM agar, germ tube tests, chlamydospore formation on cornmeal agar, sugar assimilation, and fermentation tests). Genotypic confirmation was performed by Polymerase chain reaction-restriction fragment length polymorphism (PCR-RFLP) method.

\section{Genotypic assay}

DNA extraction DNA extraction was performed by boiling lysis method [9]. Single Candida colony from a pure fresh SDA plate was picked and inoculated into $200 \mu \mathrm{l}$ of sterile milli-Q water and kept for $10 \mathrm{~min}$ in a heat block (Rivotek, India) at $100^{\circ} \mathrm{C}$. The extracted DNA after incubation at $100{ }^{\circ} \mathrm{C}$ was kept in a $-20{ }^{\circ} \mathrm{C}$ deep freezer for $10 \mathrm{~min}$ and then centrifuged at 10,000 rpm for $5 \mathrm{~min}$. Supernatant was stored at $-20{ }^{\circ} \mathrm{C}$ and used as template for PCR assay [10].

\section{Polymerase chain reaction}

PCR was performed for the Candida species targeting ITS1-5.8SrDNA-ITS2 region. The PCR mixture $(25 \mu \mathrm{l}$ reaction volume) comprised $10 \mathrm{pM}$ of Candida-ITSprimers ITS1 (5'-TCCGTAGGTGAACCTGCGG-3') and ITS4( $5^{\prime}$-TCCTCCGCTTATTGATATGC- $\left.3^{\prime}\right)$ [11], $0.4 \mathrm{mM}$ of dNTP mix, 1 unit of Taq polymerase, $2.5 \mu \mathrm{l}$ of 10X PCR buffer with $\mathrm{MgCl}_{2}$, and $2 \mu \mathrm{l}$ of DNA template. The PCR reaction was carried out in a Veriti 96 Thermal Cycler (Applied Biosystems, USA). The thermal cycling conditions included an initial denaturation at $94{ }^{\circ} \mathrm{C}$ for 3 min followed by 40 cycles at $94{ }^{\circ} \mathrm{C}$ for $20 \mathrm{~s}, 55^{\circ} \mathrm{C}$ for $30 \mathrm{~s}$ and $72{ }^{\circ} \mathrm{C}$ for $45 \mathrm{~s}$, and successively final extension at $72{ }^{\circ} \mathrm{C}$ for $5 \mathrm{~min}$. The PCR amplicons were fractionated by gel electrophoresis in $1 \%$ agarose with ethidium bromide 


\section{PCR gel picture for Candida ITS}

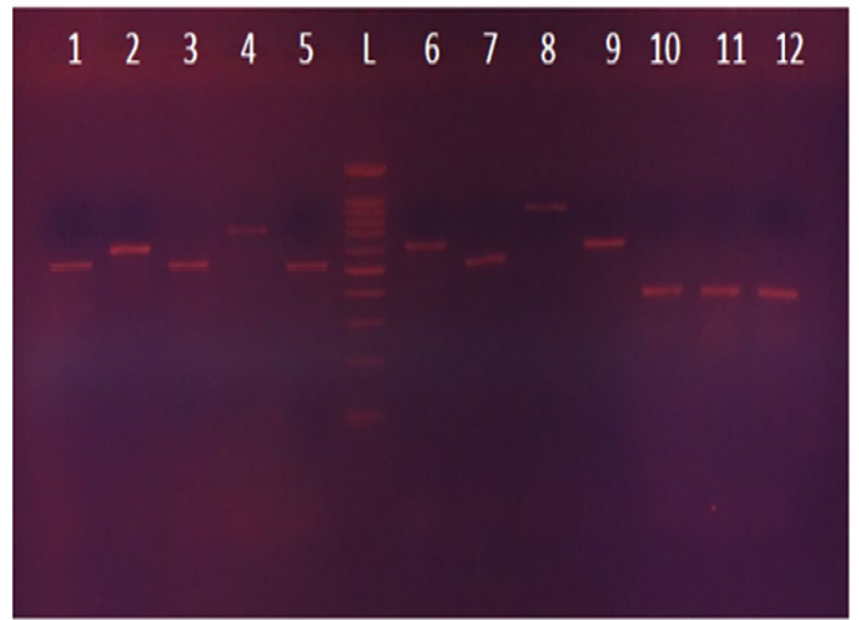

\begin{tabular}{|c|c|}
\hline LANE & ISOLATES \\
\hline 1 & A116 \\
\hline 2 & C59 \\
\hline 3 & B199 \\
\hline 4 & C78 \\
\hline 5 & B163 \\
\hline 6 & A90 \\
\hline 7 & B197 \\
\hline 8 & C75S \\
\hline 9 & B102 \\
\hline 10 & B75 \\
\hline 11 & C89 \\
\hline 12 & B73 \\
\hline
\end{tabular}

L:100bp molecular size marker,Lane1: C.albicans, Lane 2 : Pichia anomala, Lane 3: Candida tropicalis ,Lane 4: Candida kefyr Lane 5: C.krusei Lane 6: C.guilliermondii Lane 7: C.orthopsilosis Lane 8: C. glabrata Lane 9: P.anomala Lane10: C.rugosa, Lane 11: C.lusitaniae Lane 12: C.rugosa

Fig. 1 Gel electrophoresis picture showing the PCR amplicons of varied Candida species

$(0.5 \mu \mathrm{g} / \mathrm{ml})$ for $25 \mathrm{~min}$ at $135 \mathrm{~V}$ using Mupid-exU system (Takara, Japan). The gel was examined under BioGlow UV Transilluminators (Crystal Technology, USA) (Fig. 1).

\section{Restriction fragment length polymorphism}

ITS PCR product $(8.8 \mu \mathrm{l})$ was digested with $0.2 \mu \mathrm{l} \mathrm{MspI}$ $(4 \mathrm{U})$ restriction enzyme (New England Biolabs) and $1 \mu \mathrm{l}$ 10X Enzyme Buffer [11]. The restriction digestion was performed in Veriti 96 Thermal Cycler (Applied Biosystems, USA). The incubation temperature and timing for the mix was $37{ }^{\circ} \mathrm{C}$ and $60 \mathrm{~min}$ respectively. This was followed by heat inactivation at $85^{\circ} \mathrm{C}$ for $5 \mathrm{~min}$. The RFLP products were analysed by gel electrophoresis ( $2 \%$ agarose gel with $0.5 \mu \mathrm{g} / \mathrm{ml}$ ethidium bromide) and the restriction patterns were compared with in silico restriction pattern by pDRAW32 (V 1.1.140) with the sequences from NCBI (Fig. 2).

To authenticate the RFLP based identification of Candida species, representative isolates from each species were sequenced using ITS1 primer using ABI PRISM ${ }^{\circledR}$ BigDye $^{\text {TM }}$ Terminator and ABI 3730XL sequencer (Applied Biosystem, USA) at Macrogen Inc. (Seoul, Korea). Species identification of Candida was achieved by comparing the nucleotide sequence of ITS15.8SrDNA-ITS2 region against known sequences available in the GenBank database using the Basic Local Alignment Search Tool (BLAST), (https://blast.ncbi. nlm.nih.gov/Blast.cgi). The ITS gene sequences of the isolates was deposited in the NCBI GenBank database and the accession numbers obtained for C. tropicalis, C.lusitaniae, Pichia anomala formerly C.pelliculosa, Pichia anomala, C. albicans, C.lusitaniae, C.glabrata, C. guilliermondii, C.krusei and C. albicans were MN394869, MN394870, MN394871, MN39487, MN394872, MN394874, MN394875, MN394876, MN394877 and MN394878 respectively.

\section{Statistical analysis}

The significance of oral Candidal carriage was calculated using independent $\mathrm{T}$ test. Odds and risk ratio of Candida to OSCC and OPMD was calculated using Pearson's Chi square test with a confidence interval of $95 \%$. P value $<0.05$ was considered significant.

\section{Results}

Demographic details and prevalence of Candida species among the three groups is shown in the Table 1. Predominance of non albicans Candida species was observed in all three groups. Among OSCC group C. krusei (21\%), C. tropicalis (21\%), Pichia anomala (21\%), C. famata (17\%), C. glabrata (5\%), C. rugosa (6\%), C. orthopsilosis (4\%), C. keyfr (2\%), C. lusitaniae (2\%) and C. stellatoides (1\%) were the non albicans Candida species isolated. $P$. anomala formerly C. pelliculosa (33\%), C. krusei (27\%), C. tropicalis (10\%), C. famata (9\%), C. rugosa (7\%), C. guilliermondii (5\%), C. parasilopsis (3\%), C. orthopsilosis (3\%), C. glabrata (1\%), C. stellatoides (1\%), C. intermedia (1\%) were the non albicans Candida species isolated in 


\section{RFLP Gel picture for Candida ITS}

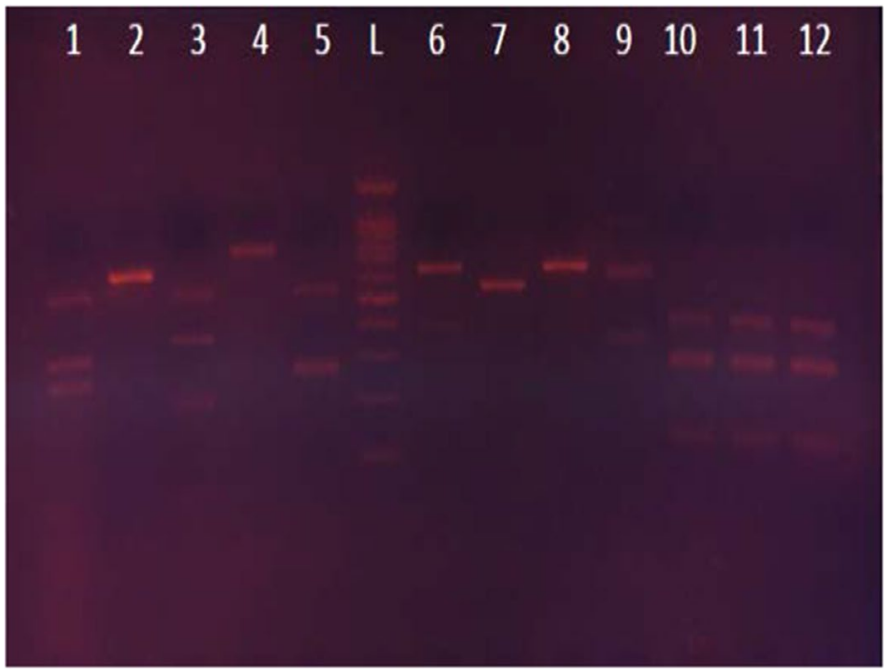

\begin{tabular}{|c|c|}
\hline LANE & ISOLATES \\
\hline 1 & A116 \\
\hline 2 & $C 55$ \\
\hline 3 & B199 \\
\hline 4 & C78 \\
\hline 5 & B163 \\
\hline 6 & A90 \\
\hline 7 & B197 \\
\hline 8 & B102 \\
\hline 9 & $C 755$ \\
\hline 10 & B75 \\
\hline 11 & $C 89$ \\
\hline 12 & B73 \\
\hline
\end{tabular}

L:100bp molecular size marker,Lane1: C.albicans, Lane 2 : Pichia anomala, Lane 3: Candida tropicalis ,Lane 4: Candida kefyr Lane 5: C.krusei Lane 6: C.guilliermondii Lane 7: C.orthopsilosis Lane 8: C. glabrata Lane 9:

P.anomala Lane10: C.rugosa, Lane 11: C.lusitaniae Lane 12: C.rugosa

Fig. 2 Gel electrophoresis picture showing the PCR fragments of restriction digestion with Mspl restriction enzyme

Table 1 Demographic details and Prevalence of Candida species in OSCC, OPMD and Control

\begin{tabular}{|c|c|c|c|c|c|c|c|c|}
\hline \multirow[t]{2}{*}{ Groups } & \multicolumn{2}{|l|}{ Sex n (\%) } & \multirow[t]{2}{*}{ Total } & \multirow[t]{2}{*}{ Age range (years) } & \multirow{2}{*}{$\begin{array}{l}\text { Oral } \\
\text { Candidal } \\
\text { Carriage (\%) }\end{array}$} & \multirow[t]{2}{*}{ C. albicans (\%) } & \multirow{2}{*}{$\begin{array}{l}\text { Non albicans } \\
\text { Candida (\%) }\end{array}$} & \multirow{2}{*}{$\begin{array}{l}\text { Presence of multiple Candida } \\
\text { species in the same subject } \\
(\%)\end{array}$} \\
\hline & Female & Male & & & & & & \\
\hline OsCC & $19(19.5)$ & $78(80.4)$ & 97 & $31-74$ & 72.2 & 42.6 & 72.1 & 14.7 \\
\hline OPMD & $11(5.5)$ & $189(94.5)$ & 200 & $24-76$ & 58 & 44.8 & 66.4 & 7.7 \\
\hline Healthy controls & $15(7.5)$ & $185(92.5)$ & 200 & $22-65$ & 20.5 & 43.5 & 69.2 & 15.4 \\
\hline
\end{tabular}

OPMD group. The non albicans Candida species from the healthy controls were $P$. anomala formerly C. pelliculosa (40\%), C. tropicalis (24\%), C. krusei (17\%), C. guilliermondii (4\%), C. parasilopsis (4\%), C. orthopsilosis (4\%), C. dubliniensis (4\%), and C. nivariensis (4\%).

Prevalence of $P$. anomala was high among health (43.5\%) followed by OPMD (33\%) and OSCC (21\%). The odds/risk ratio for OSCC and OPMD were/95\% CI 11.87, 6.69-21.05/95\% CI 4.25, 3.03-5.96 and 95\% CI $6.99,4.38-11.15 / 95 \%$ CI $3.52,2.52-4.91$ respectively. Odds ratio and Risk ratio for oral candidal carriage among OSCC \& OPMD was found to be highly significant $(\mathrm{p}=.001)$. The mean $\mathrm{cfu} / \mathrm{ml}$ was significantly high in $\operatorname{OSCC}(18601.04, \mathrm{p}=0.000)$ and OPMD (4238, $\mathrm{p}=0.001)$ compared to healthy controls (222).

\section{Discussion}

The results of the present study show a highly significant oral candidal carriage among OSCC and OPMD groups compared to healthy cohorts. Majority of the earlier studies on oral Candidal carriage in OSCC and OPMD have employed solely phenotypic assays and hence the present study focused to discuss only the literature with similar genotypic methodology. The present study reports a high prevalence of non albicans Candida in all the three groups. (Table 1) This finding was not in line with a similar study by Alnuaimi et al. and Shokohi et al. $[12,13]$. The non albicans Candida species diversity was high in OSCC and OPMD compared to healthy controls. Here, we report high species diversity in OSCC and OPMD. This may be attributed to the analysis by in silico restriction pattern by pDRAW32. Few subjects in all the three groups presented mixed colonization of Candida species in their saliva (Table 1). Interestingly, the present study by culture method identified 10 different non albicans Candida species among OSCC compared to an oral mycobiome study who have reported only three different Candida species by performing Next Generation Sequencing coupled with a species-level taxonomy assignment algorithm [14]. 
Among the non albicans Candida all the three groups showed a high prevalence of C. krusei, C. tropicalis and Pichia anomala formerly Candida pelliculosa while a similar Iranian study by Shokohi et al. reported predominance of C. glabrata, C. tropicalis and C. krusei in patients with OSCC [13]. To the best of our knowledge this may be the first study to report the presence of $P$. anomala formerly $C$. pelliculosa in all the three groups. P. anomala oral carriage was found to decrease in OSCC and OPMD conditions compared to health. This finding signifies the role of $P$. anamola in health. This is in coherence with a study on HIV patients who has reported the antagonistic effect of $P$. anamola on Candida species [15]. Candida famata was totally absent in the saliva of healthy controls. Conversely C. famata was present in the saliva of OSCC (17\%) and OPMD (9\%) patients. The present study detected C. parapsilosis, C. guilliermondii and $C$. dubliniensis whereas a similar study by Alnuaimi et al. did not report these Candida species [12]. Diverse candida species in the present study may be attributed to PCR-RFLP method of identification.

High odds and risk ratio for oral Candidal carriage among OSCC \& OPMD suggests their association to these conditions. The odds of oral Candidal carriage among OSCC in the present study are 4.25. This finding suggests Candida infection as a risk factor for OSCC which is in concurrence with a previous study [16]. Non albicans Candida was the predominant isolate in OPMD which is in contrast to several other studies who have followed phenotypic methods alone for identification of Candida species [17, 18]. In OPMD, erythroplakia and erythroleukoplakia which has high malignant transformation potential presented with high oral Candidal carriage. The Candidal carriage in OSMF was higher than leukoplakia. C. albicans was the predominant (87.5\%) species isolated in OSMF patients. Majority of the OSCC patients had documented risk factors for OSCC such as the habit of alcohol consumption, smoking and chewing of tobacco products which are to be considered for their confounding effects. However, these risk factors may have attributed in part for the high incidence of oral Candidal carriage among OSCC patients. Yet there were sizable number (13.4\%) of subjects in OSCC group who did not have any of the deleterious habits. High Oral Candidal carriage in OSCC and OPMD may be an added burden in these patients which adds on to their morbidity.

High prevalence of oral candidal carriage in patients with OSCC and OPMD suggests their association to these conditions. This finding recommends the need for their suppression to prevent opportunistic infections which may help in subsequent prevention of transformation of OPMD to OSCC. In silico restriction pattern has helped in elucidating the Candidal species diversity in OSCC \& OPMD. Non albicans Candida was the predominant group supporting its emergence in recent times. This may be the first study to report significant presence of $P$. anomala in all the three groups. The findings of the present study with high Candida diversity may be attributed to the genotypic method of identification in particular, PCR-RFLP method.

\section{Limitations}

- Samples from different geographical regions would enhance significance to the study.

- The association of Candida species to OSCC and OPMD can be further strengthened if significant number of patients with absence of deleterious habits were included.

\section{Abbreviations}

OSCC: Oral squamous cell carcinoma; OPMD: Oral potentially malignant disorders; PCR: Polymerase chain reaction; RFLP: Restriction fragment length polymorphism.

\section{Acknowledgements \\ The authors wish to thank The DST-FIST (Ref.No.SR/FST/College-23,2017) Government of India, New Delhi for utilizing the funded research equipment facilities of Sree Balaji Dental College and Hospital, Pallikaranai, Chennai,Tamil Nadu, India.}

\section{Authors' contributions}

SLS and KM wrote the proposal, KM and SLS participated in data collection and analysis VN, SLS and KM participated in the analysis, KM, SLS and VN participated in drafting, writing, reviewing of the manuscript for publication. All authors read and approved the final manuscript.

\section{Funding}

Nil.

\section{Availability of data and materials}

All data generated or analysed during this study are included in this published article [and its supplementary information files].

\section{Ethics approval and consent to participate}

The present study was approved by the institutional ethics committee of Sree Balaji Dental College and Hospital (IEC approval No: SBDCECM105/13/42). Written informed consent was obtained from all the patients willing to participate in the study.

\section{Consent to publish}

Not applicable.

\section{Competing interests}

Nil.

\section{Author details}

${ }^{1}$ Department of Oral Pathology and Microbiology, Sree Balaji Dental College and Hospital, Bharath Institute of Higher Education and Research, Chennai, Tamil Nadu 600100, India. ${ }^{2}$ Department of Microbiology, Research Lab for Oral-systemic Health, Sree Balaji Dental College and Hospital, Bharath Institute of Higher Education and Research, Chennai 600100, Tamil Nadu, India. ${ }^{3}$ Research Lab for Oral-systemic Health, Sree Balaji Dental College 
and Hospital/ImmuGenix Biosciences Pvt Ltd, No 16/2 Nattal Garden 1st Street, Perambur, Chennai, Tamil Nadu 600011, India.

Received: 30 April 2020 Accepted: 14 October 2020

Published online: 20 October 2020

\section{References}

1. Warnakulasuriya S. Global epidemiology of oral and oropharyngeal cancer. Oral Oncol. 2009;45:309-16.

2. Khan Z. An Overview of Oral Cancer in Indian Subcontinent and Recommendations to Decrease its Incidence. WebmedCentral Cancer. 2012;3(8):WMC003626. https://doi.org/10.9754/journal.wmc.2012.00362 6.

3. MohdBakri M, MohdHussaini H, RachelHolmes A, DavidCannon R, MaryRich A. Revisiting the association between candidal infection and carcinoma, particularly oral squamous cell carcinoma. J Oral Microbiol. 2010. https://doi.org/10.3402/jom.v2i0.5780.

4. Patil S. Analyzing the association between Candida prevalence, species specificity, and oral squamous cell carcinoma: a systematic review and meta-analysis. Appl Sci. 2020;10(3):1099. https://doi.org/10.3390/app10 031099.

5. Barret AW, Kinsmill VJ, Speight PM. The frequency of fungal infection in biopsies of oral mucosal lesions. Oral Dis. 1998;4:26-31.

6. Samaranayake LP. Host factors and oral candidosis. In: Samaranayake LP, McFarlane TW, editors. oral candidosis. London: Wright; 1990.

7. Oberg SG, Izutsu KT, Truelove EL. Human parotid saliva protein composition: dependence on physiological factors. Am J Physiol. 1982;242:G231-6.

8. Silva GA, Bernardi TL, Schaker PD, Menegotto M, Valente P. Rapid yeast DNA extraction by boiling and freeze-thawing without using chemical reagents and DNA purification. Braz Arch Biol Technol. 2012;55(2):319-27. https://doi.org/10.1590/s1516-89132012000200020.

9. Leena Sankari S, Mahalakshmi K, Naveen Kumar V. Chromogenic medium versus PCR-RFLP in the speciation of Candida: a comparative study. BMC Res Notes. 2019;12:681. https://doi.org/10.1186/s13104-019-4710-5.
10. Mohammadi R, Mirhendi H, Rezaei-Matehkolaei A, Ghahri M, Shidfar MR et al. Molecular identification and distribution profile of Candida species isolated from Iranian patients. Med Mycol. 2013;51(6):657-63.

11. Alnuaimi AD, Wiesenfeld D, O'Brien-Simpson NM, Reynolds EC, McCullough MJ. Oral Candida colonization in oral cancer patients and its relationship with traditional risk factors of oral cancer: a matched casecontrol study. Oral Oncol. 2015;51(2):139-45. https://doi.org/10.1016/j. oraloncology.2014.11.008

12. Shokohi T, HashemiSoteh MB, SaltanatPouri Z, Hedayati MT, Mayahi S. Identification of Candida species using PCR-RFLP in cancer patients in Iran. Indian J Med Microbiol. 2010;28(2):147-51.

13. Perera M, Al-Hebshi NN, Perera I, Ipe D, Ulett GC, et al. A dysbiotic mycobiome dominated by Candida albicans is identified within oral squamous-cell carcinomas. J Oral Microbiol. 2017;9(1):1385369. https:// doi.org/10.1080/20002297.2017.1385369.

14. Mukherjee PK, Chandra J, Retuerto M, Sikaroodi M, Brown RE, et al. Oral mycobiome analysis of HIV-infected patients: identification of Pichia as an antagonist of opportunistic fungi. PLoS Pathog. 2014;10(3):e1003996. https://doi.org/10.1371/journal.ppat.1003996.

15. Rodriguez-Archilla A, Alcaide-Salamanca MJ. Candida species detection in potentially malignant disorders of the oral mucosa: a meta-analysis. J Dent Res Rev. 2018;5:35.

16. Bansal R, Pallagatti S, Sheikh S, Aggarwal A, Gupta D, et al. Candidal species identification in malignant and potentially malignant oral lesions with antifungal resistance patterns. Contemp Clin Dent. 2018;9(Suppl2):S309-13. https://doi.org/10.4103/ccd.ccd_296_18.

17. Saigal S, Bhargava A, Mehra SK, Dakwala F. Identification of candida albicans by using different culture medias and its association in potentially malignanat and malignant lesions. Contemp Clin Dent. 2011;2(3):188-93.

18. Galle F, Colella G, Di Onofrio V, Rossiello R, Angelillo IF, Liguori G. Candida spp. in oral cancer and oral precancerous lesions. New Microbiol. 2013;36:283-8

\section{Publisher's Note}

Springer Nature remains neutral with regard to jurisdictional claims in published maps and institutional affiliations.
Ready to submit your research? Choose BMC and benefit from:

- fast, convenient online submission

- thorough peer review by experienced researchers in your field

- rapid publication on acceptance

- support for research data, including large and complex data types

- gold Open Access which fosters wider collaboration and increased citations

- maximum visibility for your research: over 100M website views per year

At $B M C$, research is always in progress.

Learn more biomedcentral.com/submissions 